\title{
Translating Developmental Neuroscience to Substance Use Prevention
}

\author{
Nathaniel R. Riggs ${ }^{1}$
}

Published online: 2 April 2015

(C) Springer International Publishing AG 2015

\begin{abstract}
Several preventive interventions have demonstrated efficacy in reducing substance use. However, opportunities exist to further improve prevention approaches. The application of recent advances in developmental neuroscience can inform the design, implementation, and evaluation of substance use prevention programs. This paper first briefly describes the developmental integration of the prefrontal cortex with emotion and motivation centers of the brain, and the implications of this process for substance use vulnerability. Discussed next are specific examples of how developmental neuroscience can inform prevention timing, development, and evaluation. Contextual considerations are then suggested including a critical role for schools in substance misuse prevention. Finally, current theoretical and methodological challenges to the translation of developmental neuroscience to substance use prevention are discussed.
\end{abstract}

Keywords Developmental neuroscience $\cdot$ Substance use prevention $\cdot$ Tobacco $\cdot$ Alcohol $\cdot$ Marijuana $\cdot$ Executive function

\section{Introduction}

Substance use during adolescence remains an issue of significant public health concern. Since 2010, prevalence rates for

This article is part of the Topical Collection on Adolescent Substance Abuse

Nathaniel R. Riggs

nathaniel.riggs@colostate.edu

1 Human Development and Family Studies, Colorado State University, 1570 Campus Delivery, Fort Collins, CO 80523, USA most substances have remained stable and high. In 2013, four in ten 12th graders reported past-month alcohol intake, one in six reported past-month cigarette use, and more than one in five reported past-month marijuana use [1]. Once initiated, progression to addiction can be rapid, particularly during adolescence, but is not inevitable [2]. Twenty-five percent of adolescents who initiate smoking have been found to lose autonomy over their use within 30 days [3]. Alcohol dependence typically progresses over a somewhat more protracted period with dependence occurring on average 3.5 years after average initiation, at around 19 years of age [4]. Although much of the health and economic burden associated with addiction emerges in adulthood [5], it is clear that substance use is typically initiated during adolescence.

Adolescent substance use prevalence rates, the rapid progression to addiction in certain individuals, and high costs associated with addiction reinforce the need for effective substance use prevention strategies. Commendable strides have been made over the past 30 years in substance use prevention efficacy [6]. A number of substance use prevention programs, such as "Raising Healthy Children" [7, 8] and "Project Northland" [9], have demonstrated empirical support (i.e., evidence-based) [6]. However, many others fail to affect meaningful change on measures directly related to substance use [6]. Additionally, even within efficacious prevention programs, there are invariably sub-groups of participants who fail to benefit [7]. Thus, significant progress remains to be made with respect to improving approaches for substance use prevention.

One strategy for systematic program improvement is to translate modifiable generative mechanisms, or mediators, of substance use prevention. Traditional models for substance use prevention have primarily been steeped in socially, cognitively, and contextually-based theories and propose, for example, that changes in attitudes toward substance use mediate 
intervention effects [10]. Increasingly, neurocognitive processes responsible for the development of self-regulation and higher-order decision-making have been identified as complementary and potentially fruitful targets for substance use prevention [11-13] and are proposed as mediators of intervention effects. However, developmental neuroscience concepts have not typically been included within more comprehensive substance use prevention approaches.

The purpose of this review is to discuss the potential for developmental neuroscience to inform substance use prevention. Discussed first will be typical neuro-developmental processes occurring during childhood and adolescence that can contribute to increased substance use vulnerability. Opportunities for the application of neuro-developmental theory to prevention implementation, design, and evaluation will then be proposed, and examples of evidence-based strategies for promoting neurocognitive function during childhood and adolescence will be provided. Finally, important socialcontextual considerations will be discussed emphasizing likely brain-by-environment interactions affecting substance use and response to prevention.

\section{Asynchrony in Brain Development: Implications for Adolescent Substance Use Vulnerability}

Regional heterogeneity in brain development exists such that the mesolimbic dopaminergic structures directly related to reward sensitivity, sensation seeking, and motivation (e.g., ventral tegmental area (VTA) and nucleus accumbens (NA)) mature earlier than systems associated with inhibitory control (e.g., prefrontal cortex) [14]. These reward-related centers of the brain then interact with hormonal changes catalyzed by puberty, and developmental changes in the social environment, such as increased importance of peer/romantic relationships relative to parents, as influences on decision-making and behavior in adolescence [15]. These developmental processes, which include heightened reward sensitivity and drive for exploration and novelty, are thought to facilitate adolescents' transition to adulthood [16] and may heighten the capacity for learning and creativity [17]. However, they also contribute to heightened vulnerability for maladaptive risk-taking behaviors including substance use $[18,19]$.

Substance use vulnerability during adolescence is exacerbated by the relatively late development of the prefrontal cortex (PFC), which is associated with higher-order meta-cognition and "top-down" self-regulation over "bottom-up" emotion- and reward-related drives. Neural white matter growth and gray matter pruning continue well into emerging adulthood, rendering the PFC the last part of the brain to achieve structural and functional maturity [20]. Heightened reward sensitivity and risk-taking in combination with protracted PFC development generate a widow from early adolescence into emerging adulthood during which "bottom up" reward- and emotion-related processing is particularly salient, but "top down" processes required to regulate strong impulses and emotions have yet to fully mature [14].

The higher-order metacognitive skills associated with PFC development are generally considered to fall under the rubric of executive function (EF). Although a universally agreed upon EF framework does not exist, three commonly referenced EF processes are inhibitory control-the capacity to inhibit a prepotent or automatic thought in favor of a more desirable or healthy response; working memory - the ability to keep multiple streams of thought "on-line" for potential mental manipulation; and cognitive flexibility - the ability to shift cognitive set or attention fluidly from one object to another [21]. Other EF processes, sometimes considered to be higher-order $\mathrm{EF}$, include planning, organization, sequencing, and task completion [22].

Research suggests that individual differences in EF, however conceptualized, are associated with risk for substance use during childhood and adolescence. To date, most of the focus in this regard has been placed on the role of inhibitory control. For example, Tarter and colleagues have demonstrated that "neurobehavioral disinhibition" assessed by interview, questionnaire, and behavioral EF tasks in early adolescence predicted moderate alcohol intake and substance use disorder in later adolescence $[23,24]$. Others have demonstrated that response disinhibition, assessed by a computerized stop signal reaction time test prior to initiation of substance use, significantly predicted later substance use disorder and alcohol misuse among high-risk youth [25]. Imaging studies appear to confirm behavioral research showing that neural indicators of EF (i.e., P300 amplitude and decreased regional blood flow to the PFC) have been linked to greater substance use during adolescence [26, 27]. Finally, Riggs and colleagues have extended this work downward to elementary school youth and demonstrated that associations between EF deficits and substance use initiation manifest as early as the fourth grade [28]. Altogether, this research illustrates that EF proficiency is negatively associated with patterns of early substance use initiation and later misuse, suggesting its potential as a target for substance use prevention. However, few attempts have been made to specifically promote neurocognitive function within the context of substance use prevention. It is argued here that research which applies developmental neuroscience concepts to substance use prevention may contribute to increased program precision and effectiveness.

\section{Applying Developmental Neuroscience to Substance Use Prevention}

Principles of developmental neuroscience can be applied to the implementation, design, and evaluation of substance use prevention. The following sections review how these principles can inform the optimal timing and content of prevention 
programs, the logic models underlying substance use prevention programs, and the evaluation of program outcomes.

\section{Prevention Timing and Developmentally Tailored}

Content Developmental neuroscience can inform prevention timing by indicating the developmental window within which mediating neurocognitive processes, such as EF, are amenable to change through behavioral intervention. Since substance use prevention programs are typically implemented during early adolescence, a period of PFC malleability, they appear to be well timed from a developmental neuroscience perspective. However, growth and development of the PFC, and associated EF processes, begins in early childhood. To the extent that an intervention could impact PFC maturation and EF processes (within biological and developmental constraints) [29], prevention strategies could be implemented during childhood. During childhood and adolescence, schools are a primary context for learning and socialization. For this reason, among others, schools are a natural context for substance use prevention, which is often administered universally to all students.

In childhood, substance use prevention programs may take the form of social-emotional learning (SEL) curricula. An overarching principle of SEL curricula is that by teaching strategies for early competence in skills with EF foundations (e.g., self-control, emotion regulation, and decision-making skills) in school [30], youth will develop the capacity to make well-regulated, healthy decisions, including those related to later substance use. Implementation of evidence-based SEL programs in childhood may provide the regulatory and decision-making foundation for later implementation of "substance use prevention programs" during adolescence.

Several registries of evidence-based SEL programs exist, including Blueprints for Healthy Youth Development (Blueprints), the National Registry of Evidence-Based Programs and Programs and Policies (NREPP), and the Office of Juvenile Justice and Delinquency Prevention Model Program Guide. However, little is known about whether implementation of SEL programs in childhood can prevent substance use in adolescence due to lack of long-term follow-up. Among the challenges to long-term follow-up is convincing funding agencies that longitudinal outcomes of childhood SEL programs will have significant policy implications for preventing adolescent substance use.

In short, a developmental neuroscience approach to the timing and content of substance misuse prevention suggests that programs can be implemented starting in childhood (e.g., pre-school), with content tailored to continuing neurocognitive maturation into adolescence. In so doing, developmentally tailored and sequenced preventive interventions that begin in childhood and continue into adolescence will have a greater capacity to alter the underlying neuro-circuitry of the brain, potentially leading to sustained prevention effects. It is under conditions of sustained intervention implementation, such as this, that prevention efforts have been shown to be most effective [31].

Including Neuro-developmental Theory in Program Logic Models Substance use prevention programs included in evidence-based registries, such as "Blueprints," typically target established mediators to substance use, such as knowledge, attitudes, and expectations regarding drugs. Fewer evidence-based programs include self-regulation and decision-making into underlying program logic models, and very few include principles of developmental neuroscience despite continued PFC development into young adulthood. Programs including neurocognitive models in an overarching prevention logic model are typically characterized as the SEL programs described earlier. The following paragraphs describe two example SEL programs that are informed by neurodevelopmental theory: Promoting Alternative THinking Strategies (PATHS; 32), and Head Start -Research Based, Developmentally Informed (Head Start REDI; 33). Two other programs, which do not specifically target $\mathrm{EF}$ improvement in the program's logic model, but which target self-regulatory processes conceptually linked to EF (e.g., drug resistance skills), are also discussed for comparison: Life Skills Training (LST; 34) and Positive Action (PA; 35).

PATHS is a pre- and elementary school curriculum based on large part upon developmental models of brain organization and EF suggesting that children often react impulsively due to still-developing top-down prefrontal cortical control over mesolimbic emotion centers of the brain [32]. As such, PATHS includes training in self-control strategies such as inhibitory control, self-talk to verbalize feelings, and the construction of effective problem solving strategies. PATHS efficacy trials have demonstrated reductions in an array of behavioral outcomes with putative relationships to substance use, including externalizing (e.g., anger and conduct problems) and internalizing (e.g., anxiety and sadness) behaviors, peer aggression, and hyperactivity [32]. Confirming the underlying neurocognitive logic model, Riggs and colleagues demonstrated that, relative to control students, second and third grade children who participated in the PATHS curriculum demonstrated enhanced inhibitory control skills as measured by performance on the Stroop task at 9-month posttest, which mediated reduced rates of both externalizing and internalizing behaviors at 1-year follow-up [33•, 34].

Head Start REDI is a second, related, study demonstrating the ability of school-based intervention to promote $\mathrm{EF}$ as a mediator to behavioral outcomes [35]. REDI was designed as an integrated model of social-emotional learning and development (a preschool version of the PATHS Curriculum) which differs from the original iteration of PATHS in that it is implemented within the existing framework of Head Start. Randomized trials conducted on Head Start REDI have demonstrated 
program effects on two measures of EF, the Dimensional Change Card Sort (DCCS) and a behavioral rating of task orientation, which in turn, partially mediated prevention effects on teacher and observer rated improvements in socialemotional development over one academic year. Both PATHS and Head Start REDI suggest the potential for translating neuro-developmental theory into school-based intervention logic models. However, the extent to which mediational findings from early social-emotional learning programs generalize to school-based substance use prevention, particularly over extended periods of longitudinal follow-up, remains unclear.

More common are prevention programs that target cognitive and affective processes theoretically linked to EF such as self-regulation, resistance skills, and/or decision-making, but which do not specifically recognize EF processes in the program logic model [36]. As a result, EF is rarely measured when evaluating the effects of these programs, and little is known with respect to the capacity of these substance misuse prevention programs to specifically promote EF during childhood and adolescence. As an example, Life Skills Training (LST) [37], which is a Blueprints "model" multicomponent preventive intervention for early adolescents, emphasizes development of substance use resistance skills within the broader context of developmentally appropriate SEL training. Among the personal skills with theoretical EF foundations targeted by LST are decision-making, problem-solving, selfcontrol of anger/frustration (e.g., inhibiting impulsive reactions), and resistance skills which likely include inhibitory control and planning/organization. Life Skills Training is one of the most researched substance use prevention programs, with over 20 years of evidence demonstrating its ability to prevent substance use initiation, problem use, and polydrug use, among other substance use outcomes up to 10 years post-intervention [10, 38]. Tests for LST program indirect effects have identified reduced risk-taking and increased refusal skills, two EF-related measures, as mediators to substance use outcomes [37].

Positive action (PA) is a second Blueprints "model" program that can be implemented from Kindergarten through early adolescence. Like LST, PA is a comprehensive preventive intervention focusing on improving students' positive thoughts, feelings, and actions related to outcomes of interest (i.e., substance non-use). Several published studies have demonstrated PA's positive effect across a diverse array of socialemotional, academic, mental health, and substance use outcomes, some sustained into high school [39]. In addition, positive longitudinal preventive effects on substance use have been shown to be completely mediated by more proximal program effects on self-reported social-emotional competence, of which one indicator was self-control [40].

In contrast to PATHS and Head Start REDI, neither LST nor PA includes EF in their overarching logic models for substance use prevention. However, many of the social-emotional skills that are targeted as mediators to substance use in LST and PA are associated with underlying EF processes, such as self-regulation and drug resistance skills [31]. Two key questions arise from this observation. The first is whether prevention effects on SEL, in programs such as LST and PA, reflect growth in often unmeasured EF processes. If so, might perceived gains in SEL be a proxy for program effects on improvement in underlying neurocognitive function? Another possibility are cascade effects whereby prevention strengthens $\mathrm{EF}$, further promoting SEL, which in turn prevents substance use. Testing such models will require further investigations into the neurocognitive foundations of SEL and substance use resistance skills, but may prove critical to systematic program improvement through specification of intervention targets.

A second is whether added value is achieved by including prevention content specifically targeting EF processes (e.g., inhibitory control, working memory, and cognitive flexibility) versus content targeting the cognitive, affective, and/or behavioral manifestations of EF (e.g., self-regulation, resistance skills, etc.). Currently, little evidence suggests that simply providing youth opportunities to practice EF skills (e.g., computerized response inhibition training) contributes to program effects generalizing beyond specific EF task performance to actual behavioral change. The limited behavioral generalizability of some "EF promotion programs" may be due to the use of training tasks that decontextualize the interpersonal nature of the behavioral learning process, which is a characteristic of opportunities to engage in substance use. As such, it may not be likely that simply promoting EF proficiency will significantly prevent substance use unless ecologically valid opportunities for youth to practice those skills are embedded within intervention content. Currently, select evidence-based interventions with (e.g., PATHS) and without (e.g., LST) a neurodevelopmental approach to selfregulation and decision-making provide youth with these opportunities. Thus, the added value of a developmental neuroscience approach to the design of substance use prevention may be to identify increasingly specific selfregulatory and decision-making processes (e.g., inhibitory control) for future program adaptation.

Neuroscientifically Informed Prevention Evaluation The field of prevention science has progressed beyond simply demonstrating that interventions work (program efficacy) to testing how interventions work (program mediation) and for whom interventions work best (program moderation). Riggs, Greenberg, and colleagues provide reviews of the capacity for neuroscience to inform the evaluation of preventive interventions [13, 31]. Few, if any published substance use prevention studies test models of EF mediation despite established literatures showing that (1) preventive interventions can promote EF, particularly among those with initial deficits [32] and (2) 
EF deficits predict future substance use [23-29]. Limited published research linking these two literatures likely reflects the stated lack of neurodevelopmental theory in overarching prevention logic models. Ultimately, however, demonstrating a mediating role for EF in prevention trials will confirm the utility of EF in substance use prevention.

Much also remains to be known with respect to whether preexisting differences in neurocognitive proficiency moderate program effects from substance use prevention. Potentially, youth with preexisting EF deficits fail to respond to substance use prevention, particularly if those deficits are severe enough to disrupt comprehension of prevention messages and/or acquisition of social or behavioral skills protecting youth from substance use. In this case, neurocognitive deficits may be a characteristic of one subgroup of program participants resistant to prevention [11]. Should this be the case, developing non-stigmatizing, tailored interventions matched to participants' pre-existing neurocognitive profile may be one complementary approach to substance use prevention.

Conversely, the content of effective substance use prevention programs, including resistance skills, emotion regulation, and decision-making skills, may provide youth with neurocognitive deficits increased opportunities to practice EF skills. Should enhanced opportunities to practice EF contribute to enhanced behavioral development relative to EFproficient peers, this would suggest that substance use prevention may be particularly effective for one group of participants at greater relative risk. It remains unclear which of these two potential moderating relationships may be the case [41, 42]. One additional possibility is that substance use prevention programs may be most beneficial for youth with EF deficits, up to the point where those deficits become severe dysfunction (e.g., traumatic brain injury and autism) [43•]. This would argue for carefully discriminating between EF deficit and EF dysfunction when exploring potential moderating effects on substance use outcomes. One exciting future area of prevention research is to identify profiles of youth more or less sensitive to prevention effects, information that can contribute not only to a better understanding of program effects, but also inform program modifications sensitive to individual differences.

\section{Considerations and Challenges in Translating Developmental Neuroscience to Prevention}

Only when taking a social-ecological approach that views brain development as embedded within, and reciprocally influenced by, important family, peer, and educational contexts can we truly understand brain development, its association with substance use, and its application to prevention science. Advantages of universal school-based prevention, relative to substance use treatment, include the capacity to prevent new cases of substance use prior to the structural and functional changes in the brain that result from misuse, the generally less expensive per-participant costs of prevention relative to treatment, and the potential prevention of related downstream health compromising behaviors (e.g., risky sexual behavior and obesity-related patterns of food intake) that share common risk factors (e.g., EF) with substance use [23, 28]. Given the advantages of substance use prevention, the following sections discuss challenges regarding evaluating the effects of neuroscience-informed prevention on $\mathrm{EF}$, the importance of targeting populations most in need to maximize prevention effects, the need to determine optimal dosing and duration of interventions, the relative effectiveness of interventions that target improving an individual's EF versus cultivating a social context conducive to health $\mathrm{EF}$, and the implications of developmental neuroscience to inform public policy relevant to substance use prevention.

Challenges in EF Assessment Universal school-based prevention research presents challenges in EF measurement related to cost of administration on a large scale and ecological validity. With regard to large scale assessment, EF measures such as the Stroop Task or Wisconsin Card Sort can be timeintensive and costly to administer since these procedures often require participants to complete the task individually. In the context of large-scale preventive intervention trials consisting of hundreds, or thousands, of study participants, these challenges can be difficult to address. More direct measures of neural function involving imaging techniques (e.g., EEG and fMRI) also can become prohibitively expensive when evaluating large-scale prevention trials, but would provide important evidence to support the neural mechanisms underlying theorized intervention effects.

Alternatively, survey-based EF assessments have been developed and are purported to assess EF in "real-life" situations [22]. Advantages of survey-based EF assessments include potentially increased ecological validity and the ability to be simultaneously administered to multiple participants. However, like all survey-based assessments, EF surveys are subject to several forms of response bias. One potential option is to use a multi-method approach that includes both survey-based assessments for all participants (including multiple informants: youth, parent, and teacher) and task-based assessments on a selected sub-population. However, the few studies that have compared the two methodologies have found surveys and task-based measures to be only modestly correlated [44]. Future studies should compare the relative validity of EF measurement methods, particularly their predictive validity vis-à-vis substance use.

Targeting Populations for Maximum Effect Among the counterarguments to universal school-based substance use prevention is that most youth will abstain from use regardless of exposure to intervention, resulting in resources being 
expended upon participants not needing intervention. However, universal prevention is often strategically implemented in social contexts considered to be of highest risk, where patterns of greater substance use and neurocognitive deficit have also been found $[45,46]$. For example, Lee and colleagues demonstrated that conditions of economic strain, particularly poverty, during childhood significantly predicted regular smoking during adulthood, and that this direct effect was mediated by self-control [45]. Raver and colleagues showed that poverty significantly predicted decreased EF as early as 4 years of age [46]. The relationship between EF and substance use may also differ by socioeconomic status. Riggs and colleagues have demonstrated that SES significantly moderated the relationship between inhibitory control and cigarette use initiation such that there was only a significant relationship between inhibitory control and smoking prevalence for early adolescents from low-SES families, not mid- to high-SES families [47]. Altogether, this pattern of results suggests that universal school-based preventive interventions that include a focus on promoting neurocognitive function may have increased reach and impact when implemented within communities most in need.

\section{Optimizing Dose and Duration of Prevention}

Programming Another challenge to translating developmental neuroscience to school-based prevention is estimating the program dosage necessary to yield large enough effects on neurocognitive function to indirectly prevent substance misuse. Evidence-based interventions shown to promote EF (i.e., PATHS and Head Start REDI) include upwards of 150 curriculum lessons, taught two to three times per week over several years, which requires a degree of buyin not always present in school administrations. However, many substance use prevention programs limit the number of curriculum lessons to fit within schools' time and commitment constraints, likely resulting in insufficient dosage to alter underlying neural function. Thus, it is critical to (1) identify the amount of intervention necessary to promote healthy EF and (2) impress upon school officials the value of sustained program implementation, while remaining sensitive to curriculum constraints and personnel needs.

\section{Intervening on the Individual Versus the Social} Environment Some debate exists with respect to whether maximal benefit would result from intervening directly with individuals, by providing increased opportunities to practice EF skills, versus a strategy focusing on the cultivation of a social context conducive to favorable EF development [48]. Some suggest that it is unlikely that individual skill development will prove successful in preventing early problem behaviors such as initiation of substance use [49]. Promoted instead is the concept of creating environments that limit risk or reduce harm such as parental monitoring, curfews, and policy changes including increased tax rates on cigarettes and alcohol to limit their attractiveness [50]. Central to the socialecological approach taken here is that comprehensive approaches which teach individual skills, including those associated with social-emotional learning and EF, within the broader context of environmental/ecological interventions is likely to have the greatest impact on reducing youth substance use [31], and leverage effects of brain-by-environment interactions in preventing substance use.

\section{Conclusion}

Substance use during adolescence remains a significant public health issue. Developmental neuroscience concepts and methods can inform the timing and content of prevention programming and also can provide opportunities to better understand potential underlying mechanisms of prevention program success and failure and to systematically improve substance use prevention. However, increased understanding of how the brain develops within important social contexts (e.g., family and school) will be required to better comprehend the conditions under which brain development contributes to risk for substance use and ultimately the development of increasingly comprehensive bio-social-contextual models of substance use prevention.

\section{Compliance with Ethics Guidelines}

Conflict of Interest Nathaniel R. Riggs declares that he has no conflict of interest.

Human and Animal Rights and Informed Consent This review article does not contain any new studies with human or animal subjects performed by the author.

\section{References}

Papers of particular interest, published recently, have been highlighted as:

- Of importance

1. Johnston LD, O'Malley PM, Bachman JG, et al. Monitoring the future national survey results on drug use, 1975-2013: Volume I, Secondary school students. Ann Arbor: Institute for Social Research, The University of Michigan; 2014.

2. Riggs NR, Chou C-P, Li C, et al. Adolescent to emerging adulthood smoking trajectories: when do smoking trajectories diverge and do they predict early adulthood nicotine dependence? Nicotine Tob Res. 2007;9:1147-54.

3. DiFranza JR, Savageau JA, Fletcher K, et al. Symptoms of tobacco dependence after brief intermittent use: the Development and 
Assessment of Nicotine Dependence in Youth-2 Study. Arch Pediatr Adolesc Med. 2007;161:704-10.

4. Sartor CE, Lynskey MT, Heath AC, et al. The role of childhood risk factors in initiation of alcohol use and progression to alcohol dependence. Addiction. 2006;102:216-25.

5. National Institute on Drug Abuse, National Institutes of Health. Understanding drug abuse and addiction. NIDA InfoFacts. Bethesda, MD; 2012. http://www.drugabuse.gov/publications/ drugfacts/understanding-drug-abuse-addiction.

6. Spoth R, Greenberg M, Turrisi R. Preventive interventions addressing underage drinking: state of the evidence and steps toward public health impact. Pediatrics. 2008;121:S311-36.

7. Brown EC, Catalano RF, Fleming CB, et al. Adolescent substance use outcomes in the Raising Healthy Children Project: a two-part latent growth curve analysis. J Consult Clin Psychol. 2005;73: 699-710.

8. Catalano RF, Mazza JJ, Harachi TW, et al. Raising healthy children through enhancing social development in elementary school: results after 1.5 years. J Sch Psychol. 2003;41:143-64.

9. Perry CL, Williams CL, Komro KA, et al. Project Northland: longterm outcomes of community action to reduce adolescent alcohol use. Health Educ Res. 2002;17:117-32.

10. Botvin GJ, Griffin KW. Life skills training: empirical findings and future directions. J Prim Prev. 2004;25:211-32.

11. Fishbein D. The importance of neurobiological research to the prevention of psychopathology. Prev Sci. 2000;1:89-106.

12. Bradshaw CP, Goldweber A, Fishbein D, et al. Infusing developmental neuroscience into school-based preventive interventions: implications and future directions. J Adolesc Health. 2012;51: S41-7.

13. Riggs NR, Greenberg MT. Neurocognition as a moderator and mediator of adolescent substance misuse prevention. Am J Drug Alcohol Abuse. 2009;35:209-13.

14. Geier CF. Adolescent cognitive control and reward processing: implications for risk taking and substance use. Horm Behav. 2013;64: 333-42.

15. Brown SA, McGue M, Maggs J, et al. A developmental perspective on alcohol and youths 16 to 20 years of age. Pediatrics. 2008;121: S290-310

16. Dahl RE. Adolescent development and the regulation of behavior and emotion: Introduction to part VIII. Ann N Y Acad Sci. 2004;1021:294-5.

17. Steinberg L. Age of opportunity: Lessons from the new science of adolescence. New York: Houghton Mifflin Harcourt; 2014.

18. Chambers RA, Taylor JR, Potenza MN. Developmental neurocircuitry of motivation in adolescence: a critical period of addiction vulnerability. Am J Psychiatry. 2003;160:1041-52.

19. Eldreth D, Hardin MG, Pavletic N, et al. Adolescent transformations of behavioral and neural processes as potential targets for prevention. Prev Sci. 2013;14:257-66.

20. Lebel C, Beaulieu C. Longitudinal development of human brain wiring continues from childhood into adulthood. J Neurosci. 2011;31:10937-47.

21. Davidson MC, Amso D, Anderson LC, et al. Development of cognitive control and executive functions from 4 to 13 years: evidence from manipulations of memory, inhibition, and task switching. Neuropsychologia. 2006;44:2037-78.

22. Guy SC, Isquith PK, Gioia GA. Behavior rating inventory of executive function-self-report version. Odessa: Psychological Assessment Resources, Inc; 2004.

23. Riggs NR, Tate EB, Ridenour TA, et al. Longitudinal associations from neurobehavioral disinhibition to adolescent risky sexual behavior in boys: direct and mediated effects through moderate alcohol consumption. J Adolesc Health. 2013;53:465-70.
24. Tarter RE, Kirisci L, Mezzich A, et al. Neurobehavioral disinhibition in childhood predicts early age at onset of substance use disorder. Am J Psychiatry. 2003;160:1078-85.

25. Nigg JT, Wong MM, Martel MM, et al. Poor response inhibition as a predictor of problem drinking and illicit drug use in adolescents at risk for alcoholism and other substance use disorders. J Am Acad Child Adolesc Psychiatry. 2006;45:468-75.

26. Heitzeg MM, Nigg JT, Yau WY, Zucker RA, Zubieta JK. Striatal dysfunction marks preexisting risk and medial prefrontal dysfunction is related to problem drinking in children of alcoholics. Biol Psychiatry. 2010;68:287-95.

27. Porjesz B, Rangaswamy M, Kamarajan C, Jones KA, Padmanabhapillai A, Begleiter $\mathrm{H}$. The utility of neurophysiological markers in the study of alcoholism. Clin Neurophysiol. 2005;11: 993-1018.

28. Riggs NR, Spruijt-Metz D, Chou C-P, et al. Relationships between executive cognitive function and lifetime substance use and obesityrelated behaviors in fourth grade youth. Child Neuropsychol. 2012;18:1-11.

29. Zelazo PD, Carlson SM, Kesek A. The development of executive function in childhood. In: Nelson C, Luciana M, editors. Handbook of developmental cognitive neuroscience. 2nd ed. Cambridge: MIT Press; 2008. p. 553-74.

30. Riggs NR, Jahromi LB, Razza RP, et al. Executive function and the promotion of social-emotional competence. J Appl Dev Psychol. 2006;27:300-9.

31. Greenberg MT, Riggs NR. Prevention and early intervention during childhood and adolescence. In: Thapar A, Pine D, Leckman J, Scott S, Snowling M, Taylor E, editors. Rutter's Child and Adolescent Psychiatry (6th Edition) (in press).

32. Greenberg MT, Kusche CA. Promoting Alternative Thinking Strategies. Blueprint for Violence Prevention (Book 10), Institute of Behavioral Sciences, University of Colorado; 1998.

33. Riggs NR, Greenberg MT, Kusché CA, et al. The mediational role of neurocognition in the behavioral outcomes of a social-emotional prevention program in elementary school students: effects of the PATHS Curriculum. Prev Sci. 2006;7:91-102

This paper is the first to confirm the underlying neurocognitive model of a social-emotional learning curriculum. In it, distal prevention effects on childhood behavior problems were mediated by proximal prevention effects on inhibitory control. Analyses presented in this paper can be used as a model for the evaluation of substance use prevention programs based on developmental neuroscience theory.

34. Riggs NR, Black DS, Ritt-Olson A. Applying neurodevelopmental theory to school-based drug misuse prevention during adolescence. New Dir Youth Dev. 2014;141:33-43.

35. Bierman KL, Nix RL, Greenberg MT, et al. Executive functions and school readiness intervention: impact, moderation, and mediation in the Head Start REDI program. Dev Psychopathol. 2008;20:821-43.

36. Botvin GJ, Griffin KW, Diaz T, et al. Smoking initiation and escalation in early adolescent girls: one-year follow-up of a schoolbased prevention intervention for minority youth. J Am Med Womens Assoc. 1999;54:139-43.

37. Beets MW, Flay BR, Vuchinich S, et al. Use of a social and character development program to prevent substance use, violent behaviors, and sexual activity among elementary school students in Hawaii. Am J Public Health. 2009;99:1438-45.

38. Griffin KW, Botvin GJ, Nichols TR. Effects of a school-based drug abuse prevention program for adolescents on HIV risk behaviors in young adulthood. Prev Sci. 2006;7:103-12.

39. Flay BR, Allred CG. Long-term effects of the positive action program. Am J Health Behav. 2003;27(Supplement 1):6-21.

40. Lewis KM, Bavarian N, Snyder FJ, et al. Direct and mediated effects of a social-emotional and character development program on adolescent substance use. Int J Emot Educ. 2013;4:56-78. 
41. Buckman JF, Bates ME, Morgenstern J. Social support and cognitive impairment in clients receiving treatment for alcohol- and druguse disorders: a replication study. J Stud Alcohol Drugs. 2008;69: 738-46.

42. Fishbein DH, Hyde C, Eldreth D, et al. Neurocognitive skills moderate urban male adolescents' responses to preventive intervention materials. Drug Alcohol Depend. 2006;82:47-60.

43. Diamond A, Lee K. Interventions shown to aid executive function development in children 4 to 12 years olds. Science. 2011;333:959-64

There is some debate in the field as to the efficacy of behavioral interventions to promote executive function. This paper provides summary of six evidence-based strategies for promoting executive function. Among them is the Promoting Alternative THinking Strategies social-emotional learning curriculum.

44. Toplak ME, Bucciarelli SM, Jain U, Tannock R. Executive functions: performance-based measures and the Behavior Rating Inventory of Executive Function (BRIEF) in adolescents with Attention Deficit/ Hyperactivity Disorder (ADHD). Child Neuropsychol. 2008;15: 53-72.
45. Lee C-T, McClernon FJ, Kollins SH, et al. Childhood economic strains in predicting substance use in emerging adulthood: mediation effects of youth self-control and parenting practices. J Pediatr Psychol. In press.

46. Raver CC, Blair C, Willoughby M. Poverty as a predictor of 4-yearolds' executive function: a new perspectives on models of differential susceptibility. Dev Psychol. 2013;49:292-304.

47. Riggs NR, Pentz MA. Socio-economic status moderates the association between inhibitory control and cigarette use prevalence in early adolescence. Manuscript submitted for publication. 2014.

48. Bryck RL, Fisher PA. Training the brain: Practical applications of neural plasticity from the intersection of cognitive neuroscience, developmental psychology, and prevention science. Am Psychol. 2012;67:87-100.

49. Steinberg L, Dahl R, Keating D, et al. The Study of developmental psychopathology in adolescence: integrating affective neuroscience with the study of context. In: Cicchetti D, Cohen D, editors. Handbook of developmental psychopathology. 2nd ed. New York: Wiley; 2006. p. 710-41.

50. Liang L, Chaloupka FJ. Differential effects of cigarette price on youth smoking intensity. Nicotine Tob Res. 2002;4:109-14. 